\title{
An update on methods for sarcopenia diagnosis: from bench to bedside
}

\author{
Andrea P. Rossi, ${ }^{1}$ Sofia Rubele, ${ }^{1}$ Alessia D’Introno, ${ }^{2}$ Elena Zoico, ${ }^{1}$ Piero Brandimarte, ${ }^{1}$ Giulia Amadio, ${ }^{1}$ \\ Nicole Nori, ${ }^{1}$ Paola Gnerre, ${ }^{3}$ Gloria Mazzali, ${ }^{1}$ Francesco Fantin, ${ }^{1}$ Mauro Zamboni ${ }^{1}$ \\ ${ }^{1}$ Geriatric Section, Department of Medicine, University of Verona; ${ }^{2}$ Geriatric Section, Department of Medicine, University of \\ Bari; ${ }^{3}$ Medicina Interna, Ospedale San Paolo, Savona, Italy
}

\begin{abstract}
Sarcopenia has been recognized as an age-related syndrome characterized by low muscle mass, low muscle strength, and low physical performance that is associated with increased likelihood of adverse outcomes including falls, fractures, hospitalization, frailty and mortality. Therefore, it is necessary to identify the condition early for applying intervention and prevention of the disastrous consequences of sarcopenia if left untreated. Clinical definition and diagnostic criteria for sarcopenia have been developed in the last years and different tools have been proposed for screening subjects with sarcopenia, evaluating the muscle mass, the muscle strength and the physical performance. In this review we analyzed the diagnostic criteria of sarcopenia and examined the current assessment tools used for the diagnosis and screening of sarcopenia.
\end{abstract}

\section{Introduction}

Aging is characterized by a progressive decrease in skeletal muscle mass, which leads to a loss of muscle strength and function. In 1989 Irwin Rosenberg proposed the term sarcopenia (from the Greek $=\sigma \alpha \rho \chi o$ muscle, $\pi \varepsilon v l \alpha=$ deficiency, poverty) to define the gradual decline in muscle mass related to age..$^{1-4}$

Some years later, Evans and Campbell ${ }^{5}$ defined sarcopenia as age-related loss in skeletal muscle mass that results in decreased strength and aerobic capacity and thus functional capacity. Baumgartner et al. using data from the New Mexico Aging Process Study, were

Correspondence: Andrea P. Rossi, Geriatric Section, Department of Medicine, Università di Verona, Ospedale Maggiore, Piazzale Stefani 1, 37126 Verona, Italy. Tel.: +39.045.8122537 - Fax: +39.045.8122043.

E-mail: andrea.rossi@hotmail.it

Key words: Sarcopenia; aging.

Conflict of interest: the authors declare no potential conflict of interest.

Received for publication: 19 February 2018.

Revision received: 26 March 2018.

Accepted for publication: 17 April 2018.

This work is licensed under a Creative Commons Attribution NonCommercial 4.0 License (CC BY-NC 4.0).

(C) Copyright A.P. Rossi et al., 2018

Licensee PAGEPress, Italy

Italian Journal of Medicine 2018; 12:97-107

doi:10.4081/itjm.2018.995 the first to propose an operational definition of sarcopenia based on measure of lean mass as assessed by dual-energy X-ray absorptiometry (DXA). Based on the measuring of appendicular skeletal muscle mass calculated as the sum of lean mass in the arms and legs divided by height squared, subjects with relative muscle mass more than two standard deviations (SD) below the sex-specific mean of a reference population consisting of 122 young subjects aged 18 to 40 years from the Rosetta Study were defined as sarcopenic. ${ }^{6}$

Janssen and colleagues proposed an alternative definition of sarcopenia based on the skeletal muscle mass index (SMI) obtained by dividing the skeletal muscle mass in $\mathrm{kg}$ to body weight compared to 100 , with the identification of two classes of sarcopenia (class I and class II, for SMI values respectively between -1 and -2 SD and $<-2$ DS than the average of a reference group of young persons included in the NHANES III). ${ }^{7}$

Together with the decrease in muscle mass and muscle strength, qualitative changes of skeletal muscle tissue occur with aging, being mainly characterized by atrophy of muscle fiber type IIa, fasttwitch ${ }^{8}$ with a progressive decline in the efficiency of the muscle (i.e., muscle strength developed for single functional unit of muscle mass). ${ }^{9}$ At the same time, the muscle atrophy is accompanied by an increase of the connective and adipose tissue within the muscle. ${ }^{10}$

The loss of muscle in the elderly is caused by multiple factors, such as the reduced synthesis of anabolic hormones, i.e. growth hormone, androgens, estrogens, and insulin-like growth factor-I, and the increase of some inflammatory cytokines especially interleukin-1 $\beta$, tumor necrosis factor- $\alpha$, and 
interleukin-6, which play a catabolic action. ${ }^{11}$ In this context, in a recent study Rossi et al. showed that higher serum levels of anti-inflammatory markers, in particular IL-4 and IL-13, may play a protective role on fat free mass (FFM) and performance maintenance in elderly subjects.

The decrease in physical activity with aging process, the insufficient protein intake and comorbidities are other key factors in the development of strength and muscle mass loss.

A rapid decline in muscle mass has been observed in older people who remain bedridden due to an illness. It has been shown that bedridden patients show an increase of urinary nitrogenous bases excretion due to an increased protein catabolism, ${ }^{12,13}$ and that healthy elderly subjects lose an average of $1 \mathrm{~kg}$ of muscle mass in 10 days of bed rest which results in $16 \%$ reduction of the isokinetic strength of the knee extensor muscles. ${ }^{14}$ This marked loss is three times greater than that observed in healthy young adults after 28 days of bed rest. ${ }^{15}$

\section{Epidemiology of sarcopenia}

The prevalence of sarcopenia varies between 7 to $50 \%$ based on the diagnostic criteria, cutoffs chosen, and methods used to measure lean body mass and muscle strength. ${ }^{16,17}$ A recent systematic review including a final sample of eighteen prevalence papers showed higher prevalence of sarcopenia in institutionalized older adults (14-33\%), compared to community dwelling elderly (1-29\%) or subjects admitted to acute care hospitals $(10 \%) .{ }^{18}$ A prevalence increase was observed with age with no significant differences between men and women.

In a recent cross-sectional observational study on 730 elderly people, a higher prevalence of sarcopenia was demonstrated in subjects with chronic diseases (heart failure, cognitive impairment, Parkinson's disease, renal failure, peripheral artery disease and hip fracture) and people with low educational level. ${ }^{19}$

\section{Health consequences of sarcopenia}

Sarcopenia is a subject of increasing interest in the scientific community for its multiple clinical and epidemiological implications. In fact, sarcopenia, which is characterized by progressive loss of skeletal muscle mass and strength, results in physical disability, reduced quality of life, and increased risk of fractures, hospitalization and mortality. ${ }^{20-24} \mathrm{~A}$ recent multicenter observational study involving 770 hospitalized patients showed a significantly higher in-hospital and 1-year mortality in participants with sarcopenia diagnosed according to EWGSOP criteria. ${ }^{25}$

\section{Definition and diagnostic criteria}

The first definition of sarcopenia was based only on skeletal muscle mass measurement by DXA or bioimpedantiometry (BIA). ${ }^{6,7,26}$

This definition of sarcopenia used in several epidemiological studies has important limitations. In fact, several studies showed that age-related decline in muscle strength is quicker and poorly correlated with the decline in muscle mass. Although muscle mass is an important determinant of muscle strength, ${ }^{27-29}$ agerelated loss of muscle mass only partially explains the loss of muscle strength. In a metanalysis Manini and Clark showed that low lean mass is generally a poor predictor of impaired physical performance, physical disability and functional limitation, outcomes more related to muscle weakness. ${ }^{30}$

Since 2010, several groups of experts and in particular the European Working Group on Sarcopenia in Older People (EWGSOP) and the International Working Group on Sarcopenia (IWG) have convened with the goal of establishing consensus diagnostic criteria for sarcopenia taking into account also its functional consequences. ${ }^{31-33}$ All the consensus groups agree in stating that a sarcopenia diagnosis should include both low muscle mass and poor muscle function as assessed by either low muscle strength or impaired physical performance.

The EWGSOP established three criteria for a diagnosis of sarcopenia: i) criterion 1: decreased muscle mass; ii) criterion 2: reduction of muscle strength; iii) criterion 3: decreased physical performance.

The documented presence of the criterion 1 associated with criterion 2 or criterion 3, allows a diagnosis of sarcopenia (Table 1). ${ }^{32}$

The EWGSOP suggests three different stages of sarcopenia which attest the severity of the condition: presarcopenia, sarcopenia, and severe sarcopenia. The presarcopenia is characterized by the loss of muscle mass not associated with strength or physical performance loss. The sarcopenia is defined by the reduction in muscle mass associated with strength or physical performance loss. The severe sarcopenia is characterized by the presence of the three diagnostic criteria.

Identifying the different stages of sarcopenia can be useful for establishing the treatment and defining appropriate recovery objectives.

More recently, in order to create a new definition of the criteria for sarcopenia validated by larger studies, it was approved The FNIH Sarcopenia Project promoted by the Foundation for the National Institutes of Health (FNIH), a public/private partnership made up of representatives of the National Institute of Aging, the National Institute of Arthritis and Musculoskeletal and Skin Diseases, the Center for 
Drug Evaluation and Research/Food and Drug Administration, several leading academic institutions and six pharmaceutical companies.

The FNIH Sarcopenia Project analyzed results from nine large population studies with longitudinal measures of muscle mass and function (Age, Gene/Environment Susceptibility-Reykjavik Study, Boston Puerto Rican Health Study, 6 clinical trials of the University of Connecticut; the Framingham Heart Study, the Health, Aging, and Body Composition Study, the Aging InChianti study; the Osteoporotic Fractures in Men Study, Rancho Bernardo Study, Study of Osteoporotic Fractures), for a total of 26,625 men and women aged 65 years and older.

The Project recently published a series of five papers $^{33-37}$ describing rationale, methods, and recommendations resulting from a year-long effort supported by the FNIH Biomarkers Consortium.

Specifically the Sarcopenia Project aimed to determine a level of muscle weakness associated with mobility disability and a degree of muscle mass that identifies muscle weakness and to evaluate whether the criteria for muscle weakness and low lean mass are associated with incident mobility impairment defined as gait speed less than $0.8 \mathrm{~m} / \mathrm{s}$. Sex-specific cut-points for handgrip strength that discriminate individuals with mobility impairment ${ }^{34}$ and cut-points for appendicular lean mass measured by DXA, that were associated with weakness defined by the previously calculated cut-points, ${ }^{35}$ were obtained.

Absolute appendicular lean mass emerged as the single best discriminator of weakness (low grip strength). Taking into account the obesity influence on the relationship between lean mass and muscle strength, alternative cut-points for appendicular lean mass standardized to body mass index (BMI) were proposed as a discriminator of weakness.

Low appendicular lean mass standardized to BMI was associated with a more modest increased risk of mobility impairment compared to low grip strength, whereas low absolute appendicular lean mass was not associated with mobility impairment. ${ }^{32}$

Among those classified as weak by the low grip strength criteria, the risk of mobility limitation did not differ between those with and without low lean mass, indicating that weakness is the primary determinant of future mobility problems. Nevertheless, the investigators noted that among those with weakness there was likely a subgroup for whom low lean mass was the underlying cause of their weakness, and these individuals could be targeted for treatment with therapies that increase muscle mass. Thus, the Sarcopenia Project recommended a set of sex-specific, empirically-derived cut-points for low absolute grip

Table 1. Different definitions of sarcopenia.

\begin{tabular}{|c|c|c|c|c|}
\hline & $\begin{array}{l}\text { European Working Group } \\
\text { on Sarcopenia in Old People } \\
\text { (EWGSOP) } \\
\text { (Cruz-Jentoft AJ) }\end{array}$ & $\begin{array}{l}\text { International working group } \\
\text { on sarcopenia (IWG) } \\
\text { (Fielding RA) }\end{array}$ & $\begin{array}{l}\text { Special interest Group: } \\
\text { Cachexia-anorexia in } \\
\text { chronic wasting disease } \\
\text { (Muscaritoli M) }\end{array}$ & $\begin{array}{l}\text { Sarcopenia with limited } \\
\text { mobility: an international } \\
\text { consensus } \\
\text { (Morley JE) }\end{array}$ \\
\hline Definition & $\begin{array}{l}\text { Syndrome characterized by } \\
\text { a progressive generalized } \\
\text { muscle strength and mass loss } \\
\text { with increate risk of } \\
\text { unfavorable events such as } \\
\text { physical disability, reduced } \\
\text { quality of life and increased } \\
\text { mortality }\end{array}$ & $\begin{array}{l}\text { The loss of muscle mass and } \\
\text { strength associated with aging }\end{array}$ & $\begin{array}{l}\text { The term sarcopenia originally } \\
\text { referred solely to the loss of } \\
\text { muscle mass with age, and } \\
\text { as such, is an almost universal } \\
\text { phenomenon } \\
\text { Current sarcopenia definitions } \\
\text { incorporate not only muscle } \\
\text { mass but also elements such } \\
\text { as strength and function }\end{array}$ & $\begin{array}{l}\text { Reduced muscle mass, with } \\
\text { limited mobility }\end{array}$ \\
\hline Criteria & $\begin{array}{l}\text { 1. Low muscle mass }+ \\
\text { 2. Low muscle strength } \\
\text { or } \\
\text { 3. Low physical performance }\end{array}$ & $\begin{array}{l}\text { 1. Low muscle mass } \\
\text { (i.e. a percentage of muscle } \\
\text { mass } 2 \text { standard deviations } \\
\text { below the mean measured in } \\
\text { young adults of the same } \\
\text { sex and ethnic background) } \\
\text { 2. Low walking speed } \\
\text { (<0.8 } \mathrm{m} / \mathrm{s} \text { over } 4 \text { meters } \\
\text { gait speed test) }\end{array}$ & $\begin{array}{l}\text { 1. Recommended cutoff for } \\
\text { weakness: grip strength } \\
\text { (GSMAX) }<26 \mathrm{~kg} \text { for males } \\
\text { and }<16 \mathrm{~kg} \text { for females } \\
\text { Alternate: grip strength } \\
\text { adjusted for BMI } \\
\text { (GSMAXBMI) }<1.0 \text { for } \\
\text { males }<0.56 \text { for females } \\
2 . \text { Recommended cutoff for } \\
\text { low muscle mass: } \\
\text { ALM adjusted for BMI } \\
\text { (ALM/BMI) }<0.789 \text { for } \\
\text { males }<0.512 \text { for females } \\
\text { Alternate: ALM }<19.75 \mathrm{~kg} \\
\text { for males }<15.02 \mathrm{~kg} \\
\text { for females }\end{array}$ & $\begin{array}{l}\text { 1. ALM corrected for height } \\
\text { squared of more than } 2 \\
\text { standard deviations below } \\
\text { that of healthy persons } \\
\text { between } 20 \text { and } 30 \text { years of } \\
\text { age of the same ethnic group } \\
2 \text {. Walking speed }<1.0 \mathrm{~m} / \mathrm{sec} \\
\text { o }<400 \mathrm{~m} \text { at the } 6 \mathrm{~min} \\
\text { walking test }\end{array}$ \\
\hline
\end{tabular}


strength and low appendicular lean mass standardized to BMI as potential criteria for clinically relevant weakness and low lean mass, respectively, in older men and women (Table 1).

\section{Qualitative changes occurring in sarcopenic skeletal muscle}

Within aging, the total number of motor units (motor neuron and muscle fibers associated) decreases with a prevalent loss of fast-twitch muscle-skeletal fibers (type IIa); the remaining fibers, both type I and type II fibers, undergo atrophy, histologically detectable with a reduction of their section area. ${ }^{38}$

The age-related decrease in the number of neurons, at cortical, medullary and peripheral levels, is an important causal factor of motor units' depletion. As an adaptation mechanism, new neuro-muscular junctions are generated between the residual nerve endings and the muscle fibers that have undergone denervation (type I and type II), generating new motor units in which a single motor neuron innervates both slow and rapid twitch muscle fibers. The result of this process is the loss of muscle efficiency (that is, of the muscle strength developed for each functional unit of muscle mass). This set of modifications determines an important deterioration in physical performance with a $30-35 \%$ decrease in muscle strength. ${ }^{39}$

Further, another morphological aspect consists in lipid infiltration both within adipocytes located between the muscle fibers and at the cytoplasmic level of the muscle cells themselves (intramyocellular fat). ${ }^{40}$

At ultrastructural level, mitochondrial dysfunction causes excessive production of reactive oxygen species causing accumulation of mitochondrial DNA damage; this can trigger apoptosis with subsequent programmed death of the entire fiber by the release of cytochrome $\mathrm{c}$ into the cytosol, or by the production of pro-apoptotic proteins..$^{41,42}$

Also, the oxidative stress that occurs into sarcoplasmic reticulum can lead to apoptosis by release of calcium in the cytoplasm with consequent activation of the effector caspases. Regarding the sarcomeric structures, in addition to a significant decrease in the total amount of myosin per single fiber, there is accumulation of post-transcriptional modifications, such as the glycation of proteins, with impairment of the correct interrelation between actin and myosin, reduction of the efficacy of the muscle contraction. ${ }^{43}$

\section{Methods for muscle mass evaluation}

Several methods have been proposed for muscle mass evaluation. Although BIA and anthropometry may be easily applicable in the clinical field, they are often considered inaccurate methods for muscle mass evaluation. ${ }^{44}$ In contrast, DXA that estimates appendicular and total body lean mass, and computed tomography (CT) which measures muscle cross sectional area are considered more reliable and reproducible methods.

Among regional measures of muscle mass, the mid arm circumference measured at the midpoint of the upper arm and recorded to the nearest $0.1 \mathrm{~cm}$ was the most frequently used. However, this method was limited by the assumption for its use, that the midarm is circular, the triceps skinfold thickness is twice the average fat diameter at the middle of the upper arm. ${ }^{44}$ Sex-specific equations have been previously proposed containing corrections for bone contributions, resulting in average intra-rater error of $7 \%$ to $8 \%$ in the calculated midarm muscle area. By using this formula in obese subjects an average $50 \%$ error was observed. ${ }^{45}$

More recently Lee et al. proposed a predictive equation using upper-arm girth adjusted for skinfold, thigh circumference corrected by anterior thigh skinfold and height, and calf circumference corrected by skinfold thickness. ${ }^{46}$

$\mathrm{SMM}=\mathrm{S}(0.00744 \mathrm{CAG} 2+0.00088 \mathrm{CTG} 2+0.00441$ CCG2) +2.4 Sex - 0.048 Age

where CAG is corrected upper-arm girth adjusted for skinfold thickness, CTG is thigh circumference corrected for anterior thigh skinfold thickness, CCG is calf circumference corrected for skinfold thickness, $\mathrm{S}$ is stature in $\mathrm{m}$, age is in years, sex is 1 for male and 0 for female, and race is -2 for Asians, 1.1 for African American, 0 for white or Hispanic.

Bioelectrical impedance analysis is widely used for assessing muscle mass. This method relies on the conduction of a low electrical current and allows measuring two parameters: resistance $(\mathrm{R})$ and reactance (Xc). Reference body composition data to validate the BIA-based measurements were derived from anthropometric assessment and CT measurements of muscle and fat area, and several equations have been proposed for muscle mass estimation. ${ }^{44}$

Roubenoff and colleagues developed the following BIA equations to quantify FFM: ${ }^{47}$

$9.1536+0.4273$ height $2 / \mathrm{R}+0.1926$ weight $+0.0667 \mathrm{Xc}$ for men

$7.7435+0.4542$ height $2 / \mathrm{R}+0.1190$ weight $+0.0455 \mathrm{Xc}$ for women

where height is in cm, age in years and R in ohm; sex: male $=1$, female $=0$.

Alternative regression equations, the most used in studies conducted in elderly populations, were 
proposed and validated in a multiethnic sample of 388 men and women aged 18 to 86 years by Janssen to estimate skeletal muscle mass (SMM): ${ }^{48}$

$$
\mathrm{SMM}=0.401(\mathrm{Ht} 2 / \mathrm{R})+3.825 \mathrm{Sex}-0.071 \mathrm{Age}+
$$$$
5.102
$$

where $\mathrm{R}$ is resistance (ohm) determined at $50 \mathrm{kHz}, \mathrm{Ht}$ is height $(\mathrm{cm})$, sex is male $=1$ and female $=0$, and age is in years.

The inhomogeneous composition of body compartments, cross-sectional areas variability, FFM hydration fraction, presence of edema and differences in conductor lengths from limbs and trunk limits the use of BIA equations from one population to another and the same equation cannot be used indifferently in populations with different age, BMI or hydration status. ${ }^{49}$ However bioelectrical impedance does not require a prolonged training and is a widely available and inexpensive instrument for routinely use.

DXA is a radiologic technique that offers the opportunity to direct measure muscle, fat and bone mass. This technique, originally developed for bone mineral content assessment, has been refined for assessment of soft tissue composition. DXA exposes the patient to a much less amount of X-ray compared with $\mathrm{CT}^{50}$

Validation studies for DXA indicate good agreement with $\mathrm{CT}$ and multicompartment models, considered as reference methods.

Second generation DXA are equipped with fanbeam technology that allows much less time to complete whole-body composition and bone density scan with improved precision..$^{51}$ Fan beam machine has been used frequently in clinical trials and also in the National Health and Nutrition Survey IV. Comparison studies, assessing body composition with DXA pencil-beam and fan-beam have found that the latter measurement overestimates lean mass with, on the counterpart, an underestimation of fat mass with differences between various hardware and software between $1 \%$ and $5 \%$ for different tissues. ${ }^{52,53}$

DXA has the advantage, as compared with BIA, that allows, not only total body, but also regional body composition assessment and in particular appendicular lean mass measurement.

Potential interference of fluid accumulation in the body should be considered even when using DXA, but to a lesser extent as compared with BIA.

Hydration status alterations, for example consumption of 1 to $2.4 \mathrm{~L}$ of water 1 hour before DXA scan could determine an increase of FFM, because of alteration of extracellular fluid volume, whilst the ingestion of small amount of water did not affect body composition measurement.

DXA is considered a reasonable alternative for muscle mass assessment compared to much more sophisticate, time-consuming and expensive techniques such as magnetic resonance imaging (MRI) and CT. DXA likely represents the most accessible technique for high quality and precise body composition assessment. The radiation exposure associated with DXA is low and highly acceptable (i.e., about $1 \mathrm{mrem}$; similar to that of a 3-day background). Nevertheless, even this low dose radiation needs to be considered as a limiting factor for routine assessment of body composition.

The main limitations of DXA reside in the instrument $\cos ^{44}$ and by the dimensions of the table of the densitometer, which is limited in size and weight and therefore may not be suitable for the evaluation of subjects with high-grade obesity.

CT scan is based on X-rays that pass through the different body tissues and are attenuated in relation to the physical density of the tissues examined. The CT method offers high quality images with clear distinction between fat and other soft tissues.

CT and MRI are considered gold standards for clinical research, although they are expensive and difficult to adopt on a large scale. ${ }^{44}$

This allows a clinician to visually and mathematically distinguish the different tissues with dedicated software and in particular at the muscle level to separate intermuscular adipose tissue from mean skeletal muscle based on the differences in attenuation characteristics. ${ }^{54}$

CT has high precision with $<1 \%$ of error and is considered the best method to measure body composition changes and the effect of life style interventions in humans.

The most important limitation for the routine use of $\mathrm{CT}$ exposes individuals to ionizing radiation (i.e., about 15 mrem, much higher than DXA) and provide a marker of muscle fat content but not a quantitative determination of fat content. ${ }^{44}$ Advantages are the reliability and the relative simplicity.

Several studies support the validity of MRI estimates to quantify skeletal muscle by comparison with the dissection in human cadavers. Mitsioupoulos et al..$^{55}$ compared the cross-sectional area measurements of skeletal muscle and subcutaneous adipose tissue determined from the entire arm and leg regions of cadavers with corresponding MRI-measured crosssectional areas and reported the correlation coefficient between the two approached units.

Limitations for the use of MRI in body composition studies are attributed to limited access of instrumentation, the higher technical complexity and costs, time required for scan acquisition (about $45 \mathrm{~min}$ ), and the inability to evaluate subjects with older models of implanted metal devices (e.g., joints, pace-makers, metallic particles in the eye, etc.). On 
the other hand, MRI shows a high agreement with the $\mathrm{CT}$ and provides similar measures. It does not involve radiation exposure and, furthermore, it also has the additional capacity of multiple slice acquisition (thus, rendering $3 \mathrm{D}$ volumetric estimates for muscle mass).

\section{Methods for muscle strength evaluation}

Different assessments have been proposed to measure muscle strength in the geriatric population.

To date, the most widely used method in clinical practice appears to be the handgrip strength dynamometry because of its simplicity, timeefficiency, affordability, and reliability. This method has been shown to reflect the overall strength status of an old subject and to be a good predictor of morbidity, hospitalization and mortality. ${ }^{56}$ Standardized conditions for the tes ${ }^{57}$ include seating the subject in a standard chair with their forearms resting flat on the armchairs; six measures should be taken, three with each arm; the highest reading of the 6 measurements is reported as the final result. According to guideline for European working group on sarcopenia in older people (EWGSOP, the Sarcopenia Working Group), low muscle strength, using cut-off point for handgrip strength at two standard deviations below the mean reference value, is defined as $<30 \mathrm{~kg}$ in men and $<20 \mathrm{~kg}$ in women. ${ }^{18}$

Since lower extremity strength and in particular muscle extensor appear to be crucial in a variety of functional tasks, knee extension strength measured by isokinetic and/or isometric dynamometry has also been proposed as tool for quantification of muscle strength in elderly. However, although the measurement is feasible in frail older people, ${ }^{58,59}$ its practical applicability seems to be limited because it is more complex and time-consuming, and so far, there are limited data in older population.

Sarcopenia is not confined to only upper or lower limb muscles but rather involves a more generalized loss of skeletal muscle mass and strength, including a decline in mass and strength of the respiratory chest muscles. ${ }^{60,61}$ The EWGSOP used peak expiratory flow (PEF) value to measure respiratory muscle strength in people without lung disorders. ${ }^{18}$ However, studies on respiratory function and sarcopenia are limited and PEF values cannot currently be recommended for use as a single parameter of muscle strength.

\section{Methods for physical performance evaluation}

Gait speed, sit-to-stand time and standing balance are the most widely used tools for the assessment of functional performance. They can be evaluated as single items, but, in clinical practice, they are more often measured within the context of the short physical performance battery (SPPB) ${ }^{62}$ The SPPB is really the most commonly employed method of assessing the ability to perform the activities of daily living in mobility-limited older adults; ${ }^{63}$ it focuses primarily on lower extremity function and includes a 4 meter walk to measure gait speed, one chair stand (followed by 5 time chair stands, if the first is successfully completed), and balance stands with the feel held in different positions for $10 \mathrm{sec}$ each. The test is easily administered in a variety of contexts or settings, and a score between 0 and 4 is assigned to each component (with a maximum of 12 points). Participants presenting a score $\leq 8$ points have been described as having a poor physical performance. ${ }^{18}$

Although the usual gait speed test measured as the pace on a 4- or 6-m course is the most popular physical function and performance test in clinical practice, and sarcopenia research and is often referred to as the new vital sign because it is a sensitive predictor of physical disability, fall risk, chronic illness and premature death and has been strongly associated with independent functioning in older adults, ${ }^{64,65}$ other independent tests used to assess physical performance include the 6-min walk distance (6MWT) or $400 \mathrm{~m}$ walk test, the stair climb power test, the timed up and go (TUG) test. ${ }^{18}$

In the TUG test subjects are asked to stand up from a standard chair (seat height between 44 and $47 \mathrm{~cm}$ ), walk a distance of $3 \mathrm{~m}$ (marked on the floor) at a comfortable pace, turn, walk back and sit down. The TUG is an easy-to-apply, fast and inexpensive test; it serves as an assessment of dynamic balance, and it has been shown to be a good predictor of sarcopenia in elderly hospitalized patients ${ }^{66}$ as well as in homedwelling elderly persons. ${ }^{67}$

Longer distance walking tests are also used as valid alternative tools to evaluate the exercise capacity at levels corresponding to efforts commonly performed by elderly during daily activities. Some versions of these tests measure either distance traveled in a set amount of time, as for the 6MWT that measures the distance an individual is able to walk in 6 minutes on a hard, flat, indoor surface, or the time to walk a set distance, such as the usual-pace $400 \mathrm{~m}$ walk. ${ }^{68}$ These tests have been shown to be good indicators of functional performance in older adults, ${ }^{68,69}$ and have been used as an outcome in clinical trials, however they only include walking ability.

The stair climb power test assesses the ability to ascend and descend a flight of stairs, as well as lower extremity strength, power, and balance. ${ }^{69}$ It may be of some use in research settings. ${ }^{18}$

Finally, jumping mechanography, a procedure that uses maximal countermovement jumps to assess 
muscular power, has been proposed as new tool to assess muscular function in older adults. ${ }^{70}$ The test is a strength measurement of muscle that incorporates the functional components assessed in traditional chair rise and gait speed testing. It has been shown that jump test variables are positively correlated with lean tissue and lower body muscle strength ${ }^{70}$ and that in older adults has excellent retest reliability that was comparable to grip strength and possibly better than SPPB and gait speed, is stable over time, and can be performed safely. ${ }^{70,71}$ However, more studies in older populations and comparison of this approach with traditional assessment tools in longitudinal interventional studies are needed.

\section{Questionnaires for sarcopenia screening}

As previously reported, the EWGSOP, as well as the International Working Group on Sarcopenia (IWGS), established three criteria necessary for the diagnosis of sarcopenia, namely decreased muscle mass, reduction of muscle strength and/or decreased physical performance. Besides the EWGSOP consensus statement on sarcopenia, a number of other definitions have been published. ${ }^{72,73}$ Therefore, the diagnostic criteria include muscle mass, gait speed and/or handgrip measurements that must be performed by trained operators and preferably in a laboratory assessment.

Since these measurements are not always available and easily performed in general practitioner, acutecare and long-term care settings, there is need for an easy-to-use and widely available pre-screening tool to identify the risk of sarcopenia. Moreover, it has been shown that the gait speed test, the first step for EWGSOP diagnosis criteria, cannot be performed in nearly half of the elderly population within previously described settings. ${ }^{74,75}$

Moreover, the prevalence of sarcopenia among the different study populations varies depending on whether IWGS or EWGSOP criteria were used, ${ }^{76}$ the variations widely explained by the use of different muscle indices. As a consequence, also in order to reduce the costs related to diagnosis, recent emphasis has been placed on the diagnosis of clinically important sarcopenia, developing tools able to identify clinical risk parameters of sarcopenia.

Malmstrom and Morley developed a 5-item questionnaire, the SARC-F, based on principal features and health consequences of sarcopenia, as described in literature. ${ }^{77-79}$

The SARC-F scale includes questions that investigate strength, assistance walking, rise from a chair, climb stairs and falls. Strength was measured by asking subjects how much difficulty they had lifting or carrying $10 \mathrm{lbs}(0=$ no difficulty, $1=$ some, and $2=\mathrm{a}$ lot or unable to do). Assistance walking was investigated by asking participants how much difficulty they had walking across a room and whether they used aids or needed help to do this $(0=$ no difficulty, $1=$ some, and $2=$ a lot of difficulty, use aids, or unable to do without help). Rise from a chair was measured by asking respondents how much difficulty they had transferring from a chair or bed and whether they used aids or needed help to do this $(0=$ no difficulty, $1=$ some, and $2=$ a lot of difficulty, use aids, or unable to do without help). Climb stairs was measured by asking respondents how much difficulty they had climbing a flight of 10 steps $(0=$ no difficulty, $1=$ some, and $2=\mathrm{a}$ lot or unable to do). Falls were scored a 2 for respondents who reported falling four or more times in the past year, 1 for respondents who reported falling 1-3 times in the past year, and 0 for those reporting no falls in the past year. The score range obtained with the SARC-F questionnaire ranges from 0 to 10 ( $0-2$ points for each item, $0=$ best score, $10=$ worst), dichotomized to represent symptomatic subjects (score $\geq 4$ ) compared to the healthy ones (score 0-3).

Malmstrom et al. investigated the utility of SARC$\mathrm{F}$ in the African American Health (AAH) study, Baltimore Longitudinal Study of Aging (BLSA) and National Health and Nutrition Examination Survey (NHANES).

The AAH study includes community-dwelling, late middle-aged African Americans aged between 4965 years old at initiation in 2000-2001 and had high level of disability. ${ }^{80,81}$ The BLSA is an ongoing survey started in 1958 on normal human aging; subjects included were healthy adults at the time of enrolment. The NHANES is a nationally representative study of approximately 5000 community-dwelling individuals.

In the AAH cohort, subjects with SARC-F score $\geq 4$ showed more deficits in the instrumental activities of daily living (IADL) than those who scored 3 or less, longer standing-time from the chair, lower grip strength, lower SPPB scores with poorer physical performance, higher probability of hospitalization and of having a gait speed $<0.8 \mathrm{~m} / \mathrm{s} .{ }^{82}$ SARC-F score $\geq 4$ in BLSA was associated with higher IADL deficits, worse grip strength (at both right and left hands) and mortality in follow-up (39.4\% for SARC-F $\geq 4$ vs $8.0 \%$ in SARC-F <4). NHANES participants with SARC-F scores $\geq 4$ had lower walking speeds (slower time to walk $20 \mathrm{ft}$ ), lower strength (knee extension) and were more likely to have been hospitalized recently in cross-sectional analysis.

Cao et al. showed that SARC-F was associated with poor physical performance, grip strength and hospitalization in the previous 2 years. ${ }^{83}$ In this study, two hundred and thirty Chinese people over 65 years of age were assessed by the SARC-F scale, physical self-maintenance scale (PSMS), ${ }^{84}$ IADL, and the 
shortened version of the falls efficacy scaleinternational (the short FES-I) ${ }^{85}$ Physical performance and strength were measured respectively with the SPPB and handgrip test; hospitalization was also investigated. Poor physical performance and grip strength were independently associated with a SARCF score $\geq 4$. The short FES-I was correlated to SARC-F, poor PSMS and IADL scores were associated with SARC-F $\geq 4$ and SARC-F $\geq 4$ was associated with hospitalization in the past 2 years.

Woo et al. conducted a validation study of SARCF on a cohort of 4000 Chinese community residents aged 65 and over ${ }^{86}$ SARC-F has been validated and compared with the three definitions of sarcopenia for the European consensus groups (EWGSOP), Asian (AWGS) and the international group (IWGS); the ability of all four diagnostic tools to predict physical limitation at four years of follow-up, walking speed and ability to stand up from a chair was compared. Sarcopenic subjects according to SARC-F, EWGSOP, IWGS and AWGS all showed increased risk of physical limitations and poor physical performance at follow-up, both men and women. The SARC-F questionnaire showed a high specificity (94-99\%) and high negative predictive value, but low sensitivity ( $4.2 \%$ men and $9.9 \%$ women) for sarcopenia by using the EWGSOP criteria.

More recently Rossi et al. developed the Mini Sarcopenia Risk Assessment (MSRA) questionnaire as a prescreening tool for assessing the risk of sarcopenia. ${ }^{87}$

The questionnaire is composed of seven items and investigates anamnestic and nutritional characteristics related to the risk of occurrence of sarcopenia; four questions about general assessment (age $\geq 70$ or $<70$ years, physical activity level as walking more or less than 1000 meters, number of hospitalizations in the previous year and weight loss), and three about nutritional status (consumption of proteins and dairy products, number of meals per day). These questions reflect risk factors of muscle mass and strength loss as shown in literature.

MRSA was used in a post-hoc observational study of a community-aged population of 274 subjects, 177 women and 97 men, aged 66-78 years. According to the EWGSOP diagnostic criteria, the subjects of the study were classified as sarcopenic and not sarcopenic.

The results showed that $33.5 \%$ of the study population was classified as sarcopenic. Using the form of MRSA extended to 7-item, subjects with a score equal to or less than 30 had a 4-fold higher risk of being sarcopenic than subjects with a score greater than 30 , taken as a cut-off value [odds ratio (OR): $4.20 ; 95 \%$ confidence interval (CI): 2.26-8.06]; the area under the ROC curve was 0.786 (95\% CI: 0.725-0.847).

In a logistic regression, considering the probability of being sarcopenic as the dependent variable and the variables corresponding to the 7 items of the questionnaire as independent, two items (number of meals and consumption of milk and dairy products) showed not significant diagnostic power. A cut-off score for the 5-item questionnaire was then obtained, where the area under the ROC curve was 0.789 (95\% CI: 0.728 to 0.851 ).

Taking into account the cost of false positives and false negatives and the prevalence of sarcopenia, the optimal threshold of the original MSRA score based on 7 items is therefore 30 , with a sensitivity of 0.804 and a specificity of 0.505 , while the threshold optimal of the MSRA score based on 5 items is 45 , with a sensitivity of 0.804 and a specificity of 0.604 .

In this preliminary study, the MSRA questionnaire was predictive of sarcopenia and may be suggested as a pre-screening tool to detect this condition. In particular, the use of the short form of the MSRA questionnaire improves the ability to identify sarcopenic subjects, for sensitivity and specificity values (Tables $2^{77}$ and $3^{87}$ ).

\section{Conclusions and perspectives}

The research in the field of sarcopenia has progressed significantly over the past decade, but yet it continues to lag behind the clinical assessment, treatment, and outcomes that have been wellestablished in the field of osteoporosis. With the EWGSOP and IWG consensus and the ongoing FNIH activities in the Biomarkers Consortium, further refinements of sarcopenia criteria are expected.

The validation of the MSRA and SARC-F questionnaires should be part of further prospective or retrospective studies in wider populations. The MSRA and SARC-F questionnaires are designed to provide an inexpensive and easy-to-use tool capable of capturing the key features of sarcopenia and rules out subjects unlikely to be sarcopenic. They have the advantage that can be compiled also by the patient or by the caregiver and are applicable in postal screening, phone calls or general practitioners settings as well, thus allowing the screening for sarcopenia in outpatients.

While further studies are needed to provide a full evidence-based guidance, the management and assessment of sarcopenia is very important in the mainstream of clinical care to ensure a reduction in age-related falls, fractures, and disability and to guarantee a better quality of life with aging.

\section{References}

1. Rosenberg IH. Summary comments: epidemiological and methodological problems in determining nutritional 
status of older person. Am J Clin Nutr 1989; 50:1231-3.

2. Rosenberg IH. Sarcopenia: origins and clinical relevance. J Nutr 1997;127:990-1.

3. Barazzoni R, Nair KS. Session on protein metabolism in the elderly. Sarcopenia of the elderly. Diab Nutr Metab 2000;13:92-8.

4. Roubenoff R, Hughes VA. Sarcopenia: current concepts. J Gerontol A Biol Sci Med Sci 2000;12:716-24.

5. Evans WJ, Campbell WW. Sarcopenia and age-related changes in body composition and functional capacity. $\mathrm{J}$ Nutr 1993;123:465-8.

6. Baumgartner RN, Koehler KM, Gallagher D, et al. Epidemiology of sarcopenia among the elderly in New Mexico. Am J Epidemiol 1998;147:755-63.

7. Janssen I, Heymsfield SB, Ross R. Low relative skeletal muscle mass (Sarcopenia) in older persons is associated with functional impairment and physical disability. JAGS 2002;50:889-96.

8. Lexell J. Human aging, muscle mass, and fiber type composition (review). J Gerontol A Biol Sci Med Sci 1995;50:11-6.

9. Lindle RS, Metter EJ, Linch NA, et al. Age and gender comparisons of muscle strenght in 654 women and men aged 20-93 years. J Appl Physiol 1997;83:1581-7.

10. Rice CL, Cunningham DA, Paterson DH, Lefcoe MS. Arm and leg composition determined by computed tomografhy in young and elderly men. Clin Physiol 1989;9:207-20.

11. Morley JE, Baumgartmer RN, Roubenoff R, et al. From the Chicago Meetings. Sarcopenia. J Lab Clin Med 201;137:231-43.

12. Deitrick JE, Whedon GD, Shorr E. Effects of immobilization upon various metabolic and physiologic functions of normal men. Am J Med 1948;4:3-36.

13. Ferrando AA, Tipton KD, Bamman MM, Wolfe RR. Resistance exercise maintains skeletal muscle protein synthesis during bed rest. J Appl Physiol 1997; 82:807-10.

14. Kortebein P, Ferrando A, Lombeida J, et al. Effect of 10 days bed rest on skeletal muscle in healthy older adults. JAMA. 2007;297:1772-4.

15. Paddon-Jones D, Sheffield-Moore M, Urban RJ, et al. Essential amino acid and carbohydrate supplementation ameliorates muscle protein loss in humans during 28 days bedrest. J Clin Endocrinol Metab 2004;89:4351-8.

16. Bijlsma AY, Meskers CG, Ling $\mathrm{CH}$, et al. Defining sarcopenia: the impact of different diagnostic criteria on the presence of sarcopenia in a large middle aged cohort. Age 2013;35:871-81.

17. Batsis JA, Barre LK, Mackenzie TA, et al. Variation in the prevalence of sarcopenia and sarcopenic obesity in older adults associated with different research definitions: Dual X-Ray Absorptiometry data from the National Health and Nutrition Examination Survey 1999-2004. J Am Geriatr Soc 2013;61:974-80.

18. Cruz-Jentoft AJ, Landi F, Schneider SM, et al. Prevalence of and interventions for sarcopenia in ageing adults: a systematic review. Report of the International Sarcopenia Initiative (EWGSOP and IWGS). Age Ageing 2014;43:748-59.

19. Volpato S, Bianchi L, Cherubini A, et al. Prevalence and clinical correlates of sarcopenia in community-dwelling older people: application of the EWGSOP definition and diagnostic algoritm. J Gerontol A Biol Sci Med Sci 2014;69:438-46.

20. Goodpaster BH, Park SW, Harris TB, et al. The loss of skeletal muscle strength, mass, and quality in older adults: the health, aging and body composition study. J Gerontol A Biol Sci Med Sci 2006;61:1059-64.

21. Abellan van Kan G. Epidemiology and consequences of sarcopenia. J Nutr Health Aging 2009;13:708-12.

22. Janssen I, Baumgartner RN, Ross R, et al. Skeletal muscle cutpoints associated with elevated physical disability risk in older men and women. Am J Epidemiol 2004;159:413-21.

23. Rossi AP, Fantin F, Abete P, et al. Association between hospitalization related outcomes, dynapenia and body mass index: the Glisten Study. Eur J Clin Nutr 2018 [In press].

24. Gariballa S, Alessa A. Sarcopenia: prevalence and prognostic significance in hospitalized patients. Clin Nutr 2013;32:772-6.

25. Vetrano DL, Landi F, Volpato S, et al. Association of sarcopenia with short- and long-term mortality in older adults admitted to acute care wards: results from the CRIME study. J Gerontol A Biol Sci Med Sci 2014; 69:1154-61.

26. Newman AB, Kupelian V, Visser M, et al. Sarcopenia: alternative definitions and associations with lower extremity function. Health ABC Study Investigators. J Am Geriatr Soc 2003;51:1602-9.

27. Delmonico MJ, Harris TB, Visser M, et al. Longitudinal study of muscle strength, quality, and adipose tissue infiltration. Am J Clin Nutr 2009;90:1579-85.

28. Reed RL, Pearlmutter L, Yochum K, et al. The relationship between muscle mass and muscle strength in the elderly. J Am Geriatr Soc 1991;39:555-61.

29. Hughes VA, Frontera WR, Wood M, et al. Longitudinal muscle strength changes in older adults: influence of muscle mass, physical activity, and health. J Gerontol A Biol Sci Med Sci 2001;56:209-17.

30. Manini TM, Clark BC. Dynapenia and aging: an update. J Gerontol A Biol Sci Med Sci 2012;67:28-40.

31. Fielding R, Vellas B, Evans WJ, et al. Sarcopenia: an undiagnosed condition in older adults. Current consensus definition: prevalence, etiology, and consequences. International working group on sarcopenia. J Am Med Dir Assoc 2011;12:249-56.

32. Crutz-Jentoft AJ, Baeyens JP, Bauer JM, et al. Sarcopenia: European consensus on definition and diagnosis. Report of the European Working Group on Sarcopenia in Older People. Age Aging 2010;39:421-3.

33. Studenski SA, Peters KW, Alley DE, et al. The FNIH sarcopenia project: rationale, study description, conference recommendations, and final estimates. J Gerontol A Biol Sci Med Sci 2014;69:547-58.

34. Alley DE. Grip strength cutpoints for the identification of clinically relevant weakness. J Gerontol A Biol Sci Med Sci 2014;69:559-66.

35. Cawthon PM. Cutpoints for low appendicular lean mass that identify older adults with clinically significant weakness. J Gerontol A Biol Sci Med Sci 2014;69:567-75.

36. McLean RR. Criteria for clinically relevant weakness and low lean mass and their longitudinal association 
with incident mobility impairment and mortality: the foundation for the National Institutes of Health (FNIH) sarcopenia project. J Gerontol A Biol Sci Med Sci 2014; 69:576-83.

37. Dam TT. An evidence-based comparison of operational criteria for the presence of sarcopenia. J Gerontol A Biol Sci Med Sci 2014;69:584-90.

38. Lexell J, Downham DY. The occurrence of fibre-type grouping in healthy human muscle: a quantitative study of cross- sections of whole vastus lateralis from men between 15 and 83 years. Acta Neuropathol 1991;81: 377-81.

39. Vandervoort AA. Aging of the human neuromuscular system. Muscle Nerve 2002;25:17-25.

40. Kirkland JL, Tchkonia T, Pirtskhalava T, et al. Adipogenesis and aging: does aging make fat go MAD? Exp Gerontol 2002;37:757-67.

41. Hepple RT. Mitochondrial involvement and impact in aging skeletal muscle. Front Aging Neurosci 2014; 6:211.

42. Dirks AJ, Leeuwenburgh C. The role of apoptosis in age-related skeletal muscle atrophy. Sports Med 2005;35:473-83.

43. D'Antona G, Pellegrino MA, Adami R, et al. The effect of ageing and immo- bilization on structure and function of human skeletal muscle fibres. J Physiol 2003; 552:499-511.

44. Rossi AP, Harris TB, Fantin F, et al. The multidomain mobility lab in older persons: from bench to bedside. The assessment of body composition in older persons at risk of mobility limitations. Curr Pharm Des 2014; 20:3245-55.

45. Heymsfiled SB, Olafson RP, Kutner MH, Nixon DW. A radiographic method of quantifiying protein-calorie undernutrition. Am J Clin Nutr 1979;32:693-702.

46. Lee RC, Wang Z, Heo M, et al. Total body skeletal muscle mass: development and cross-validation of anthropometric prediction models. Am J Clin Nutr 2000;72:796-803.

47. Roubenoff R, Kehayias J, Rosemberg IH, et al. Standardization of nomenclature of body composition in weight loss. Am J Clin Nutr 1997;66:192-6.

48. Janssen I, Heymsfield SB, Baumgartner RN, Ross R. Estimation of skeletal muscle mass by bioelectrical impedance analysis. J Appl Physiol 2000;89:465-71.

49. National Institutes of Health Technology Assessment Conference Statement. Bioelectrical impedance analysis in body composition measurement. Am J Clin Nutr 1996;64:524-32.

50. Kellie SE. Measurement of bone density with dualenergy x-ray absorptiometry (DXA). J Am Med Ass 1992;267:286-94.

51. Nord RH. Evaluation of a new instrument for measuring body composition. Ann NY Med Acc Sci 2000;904:118-25.

52. Visser M, Fuerst T, Lang T, et al. Validity of fan-beam dual-energy X-ray absorptiometry for measuring fat-free mass and leg muscle mass. Health, aging, and body compositon working group. J Appl Physiol 1999;87: 1513-20.

53. Tothill P, Hannan WJ. Comparson between Hologic QDR 1000W, QDR 4500A, and Luner Expert dual energy X-ray absorptiometry scanners used for measuring total body bone and soft tissue. Ann N Y Acad Sci 2000;904:63-71.

54. Goodpaster BH, Thaete FL, Simoneau JA, Kelley DE. Subcutaneous abdominal fat and thigh muscle composition predicts insulin sensitivity independently of visceral fat. Diabetes 1997;46:1579-85.

55. Mitsiopoulos N, Baumgartner RN, Heymsfield SB, et al. Cadaver validation of skeletal muscle measurements by magnetic resonance imaging and computer tomography. J Appl Physiol 1998;85:115-22.

56. Legrand D, Adriaensen W, Vaes B, et al. The relationship between grip strength and muscle mass (MM), inflammatory biomarkers and physical performance in community-dwelling very old persons. Archiv Gerontol Geriatr 2013;57:345-51.

57. Roberts HC. A review of the measurement of grip strength in clinical and epidemiological studies: towards a standardised approach. Age Ageing 2011;40:423-9.

58. Brown M, Sinacore DR, Binder EF, Kohrt WM. Physical and performance measures for the identification of mild to moderate frailty. J Gerontol A Biol Sci Med Sci 2000;55:350-5.

59. Callahan D, Phillips E, Carabello R, et al. Assessment of lower extremity muscle power in functionally-limited elders. Aging Clin Exp Res 2007;19:194-9.

60. Ro HJ, Kim DK, Lee SY, et al. Relationship between respiratory muscle strength and conventional sarcopenic indices in young adults: a preliminary study. Ann Rehabil Med 2015;39:880-7.

61. Kera T, Kawai H, Hirano H, et al. Relationships among peak expiratory flow rate, body composition, physical function, and sarcopenia in community-dwelling older adults. Aging Clin Exp Res 2017 [Epub ahead of print].

62. Guralnik JM, Simonsick EM, Ferrucci L, et al. A short physical performance battery assessing lower extremity function: association with self-reported disability and prediction of mortality and nursing home admission. J Gerontol 1994 Mar;49:85-94.

63. Guralnik JM, Ferrucci L, Pieper CF, et al. Lower extremity function and subsequent disability: consistency across studies, predictive models, and value of gait speed alone compared with the short physical performance battery. J Gerontol A Biol Sci Med Sci 2000;55:221-31.

64. Cesari M, Kritchevsky SB, Penninx BW, et al. Prognostic value of usual gait speed in well-functioning older people-results from the Health, Aging and Body Composition Study. J Am Geriatr Soc 2005;53:1675-80.

65. Abellan van Kan G, Rolland Y, Andrieu S, et al. Gait speed at usual pace as a predictor of adverse outcomes in community-dwelling older people an International Academy on Nutrition and Aging (IANA) Task Force. J Nutr Health Aging 2009;13:881-9.

66. Prata Martinez B, Barboza Gomes I, Santana de Oliveira C, et al. Accuracy of the timed up and go test for predicting sarcopenia in elderly hospitalized patients. Clinics 2015;70:369-72.

67. Alexandre TS, Meira DM, Rico NC, Mizuta SK. Accuracy of timed up and go test for screening risk of falls among community-dwelling elderly. Rev Bras Fisioter 2012;16:381-8.

68. Newman AB, Simonsick EM, Naydeck BL, et al. Association of long-distance corridor walk performance 
with mortality, cardiovascular disease, mobility limitation, and disability. JAMA 2006;295:2018-26.

69. Bean JF, Kiely DK, LaRose S, et al. Is stair climb power a clinically relevant measure of leg power impairments in at-risk older adults? Arch Phys Med Rehabil 2007; 88:604-9.

70. Buehring B, Krueger D, Binkley N. Jumping mechanography: a potential tool for sarcopenia evaluation in older individuals. J Clin Densitom 2010; 13:283-91.

71. Hannam K, Hartley A, Clark EM, et al. Reproducibility of jumping mechanography and traditional measures of physical and muscle function in older adults. J Musculoskelet Neuronal Interact 2017;17:246-57.

72. Dam TT, Peters KW, Fragala M, et al. An evidencebased comparison of operational criteria for the presence of sarcopenia. J Gerontol A Biol Sci Med Sci 2014; 69:584-90.

73. Chen LK, Liu LK, Woo J, et al. Sarcopenia in Asia: consensus report of the Asian Working Group for Sarcopenia. J Am Med Dir Assoc 2014;15:95-101.

74. Rossi AP, Fantin F, Micciolo R, Bertocchi M, Bertassello P, Zanandrea V, Zivelonghi A, Bissoli L, Zamboni M. Identifying sarcopenia in acute care setting patients. J Am Med Dir Assoc 2014;15:7-12.

75. Landi F, Liperoti R, Fusco D, et al. Prevalence and risk factors of sarcopenia among nursing home older residents. J Gerontol A Biol Sci Med Sci 2012;67:48-55.

76. Lee WJ, Liu LK, Peng LN, et al. Comparisons of sarcopenia defined by IWGS and EWGSOP criteria among older people: Results from the I-Lan longitudinal aging study. J Am Med Dir Assoc 2013;14:528.

77. Malmstrom TK, Morley JE. SARC-F: A simple questionnaire to rapidly diagnose sarcopenia. J Am Med Dir Assoc 2013;14:531-2.

78. Cruz-Jentoft AJ, Baeyens JP, Bauer JM, et al. Sarcopenia: European consensus on definition and diagnosis: report of the European Working Group on Sarcopenia in Older People. Age Ageing 2010;39:412-3.

79. Fielding RA, Vellas B, Evans WJ, et al. Sarcopenia: an undiagnosed condition in older adults. Current consensus definition: prevalence, etiology, and consequences. International Working Group on Sarcopenia. J Am Med Dir Assoc 2011;12:249-56.

80. Morley JE, Malmstrom TK, Miller DK. A simple frailty questionnaire (FRAIL) predicts outcomes in middle aged African Americans. J Nutr Health Aging 2012; 16:601-8.

81. Miller DK, Wolinsky FD, Malmstrom TK, et al. Inner city, middleaged African Americans have excess frank and subclinical disability. J Gerontol A Biol Sci Med Sci 2005;60:207-12.

82. Malmstrom TK, Miller DK, Simonsick WM, et al. SARC-F: a symptom score to predict persons with sarcopenia at risk for poor functional outcomes. J Cachexia Muscle Wasting 2016;7:28-36.

83. Cao L, Chen S, Zou C, et al. A pilot study of the SARC$\mathrm{F}$ scale on screening sarcopenia and physical disability in the Chinese older people. J Nutr Health Aging 2014; 18:277-83.

84. Lawton MP, Brody EM. Assessment of older people: self-maintaining and instrumental activities of daily living. Gerontologist 1969;9:179-86.

85. Kempen GL. The Short FES-I: a shortened version of the falls efficacy scale-international to assess fear of falling. Age Ageing 2008;37:45-50.

86. Woo J, Leung J, Morley JE. Validating the SARC-F: a suitable community screening tool for sarcopenia? J Am Med Dir Assoc 2014;15:630-4.

87. Rossi AP, Micciolo R, Rubele S, et al. Assessing the risk of sarcopenia in the elderly: the mini sarcopenia risk assessment (MSRA) questionnaire. J Nutr Health Aging 2017;21:743-49. 